\title{
Perfil del cuidador primario en pacientes diabéticos en dos comunidades*
}

\author{
Profile of the primary caregiver for diabetic patients in two communities \\ Perfil do cuidador primário em pacientes diabéticos em duas comunidades
}

\author{
María Susana González Velázquez ${ }^{\mathrm{a}}$ \\ Universidad Nacional Autónoma de México, México \\ vinculacion.planeacion@zaragoza.unam.mx \\ ORCID: https://orcid.org/0000-0002-5851-4886 \\ Silvia Crespo Knopfler \\ Universidad Nacional Autónoma de México, México \\ ORCID: https://orcid.org/0000-0002-7720-7187
}

DOI: https://doi.org/10.11144/Javeriana.ie22.pcpd

Recibido: 29 Enero 2019

Aceptado: 12 Diciembre 2019

Publicado: 22 Julio 2020

\section{Resumen:}

Introducción: Las enfermedades de carácter crónico se han convertido en una de las preocupaciones más importantes de la sociedad moderna, debido a que significa una estadía permanente en los sistemas de salud, al tiempo que implican que un tercero se haga cargo del enfermo. Objetivo: Describir el perfil del cuidador primario del paciente diabético en dos comunidades mexicanas. Método: Estudio transversal descriptivo de agosto de 2017 a noviembre de 2018 con 38 cuidadores primarios de personas que padecen diabetes mellitus tipo 2, provenientes de dos clínicas universitarias de atención a la salud (CUAS). En la CUAS Zaragoza participaron 17 cuidadores primarios, y en la CUAS Reforma, 21. Se aplicó el instrumento denominado Valoración para el cuidador primario. Resultados: La edad promedio de los cuidadores es de 60 años \pm 15 años. El género predominante es el femenino. La mayoría tiene estudios de primaria y el $68 \%$ padece alguna enfermedad. El parentesco que predominó con los pacientes son esposas(os). El 73 \% de los cuidadores cuenta con apoyo de sus familiares. El $55 \%$ tiene un conocimiento regular sobre la diabetes mellitus y en los valores del cuidador predominó tenerle respeto a su paciente. Conclusiones: Conocer el perfil de los cuidadores permitirá a los profesionales de enfermería establecer diferentes estrategias de salud que les permita reforzar el conocimiento acerca de la enfermedad y así mejorar el estilo de vida de los pacientes para brindarles apoyo, dado el impacto y el desgaste que genera ser cuidador primario en una enfermedad.

Palabras clave: cuidadores primarios, cuidador, enfermería, diabetes mellitus tipo 2.

\section{Abstract:}

Introduction: Chronic diseases have become one of the most important concerns in modern society given that it means a permanent stay in the healthcare system for patients, while implying that a third party needs to take care of them. Objective: To describe the profile of the primary caregiver for diabetic patients in two Mexican communities. Method: Descriptive crosssectional study carried out from August 2017 to November 2018 with 38 primary caregivers of people with type 2 diabetes mellitus from two university healthcare clinics (CUAS). Seventeen primary caregivers from the CUAS Zaragoza and 21 from the CUAS Reforma took part of the study. The instrument applied was called Assessment for the Primary Caregiver. Results: The average age of the caregivers is 60 years \pm 15 years. The prevailing gender is female. Most caregivers have elementary school education and $68 \%$ have a disease. The prevailing relationship with the patients is spouses; $73 \%$ of caregivers have the support of their relatives; $55 \%$ have an average knowledge about diabetes mellitus and the prevalent caregiver's value was to respect their patient. Conclusions: Knowing the profile of caregivers will allow nursing professionals to propose different health strategies that will allow them to improve caregiver's knowledge about this disease and thus improve the lifestyle of patients and provide them with support, given the impact and wear that generates being a primary caregiver for an ill patient.

Keywords: primary caregivers, caregiver, nursing, type 2 diabetes mellitus.

\section{Resumo:}

Introdução: As doenças de carácter crônico tornaram-se uma das preocupações mais importantes da sociedade moderna, porque elas significarem uma estada permanente nos sistemas de saúde, enquanto o que implica que um terceiro cuide do doente. Objetivo: Descrever o perfil do cuidador primário do paciente diabético em duas comunidades mexicanas. Método: Estudo transversal descritivo de agosto de 2017 para novembro de 2018 com 38 cuidadores primários de pessoas com diabete mellitus tipo 2, a

Notas de autor

\footnotetext{
a Autora de correspondencia. Correo electrónico: vinculacion.planeacion@zaragoza.unam.mx
} 
partir de duas clínicas universitárias de atenção à saúde (CUAS). Na CUAS Zaragoza participaram 17 cuidadores primários e na CUAS Reforma, 21. O instrumento nomeado de Valoração para o cuidador primário foi aplicado. Resultados: A idade média dos cuidadores é de 60 anos \pm 15 anos. O gênero predominante é o feminino. A maioria tem ensino fundamental e $68 \%$ têm alguma doença. O parentesco que predominou com os pacientes é casal. $73 \%$ dos cuidadores tem apoio dos seus familiares. $55 \%$ tem conhecimento comum sobre a diabetes mellitus e nos valores do cuidador predominou ter respeito ao paciente. Conclusões: Conhecer o perfil dos cuidadores permitira aos profissionais de enfermagem estabelecerem diferentes estratégias de saúde que lhes permitirá reforçar o conhecimento acerca da doença e assim melhorar o estilo de vida dos pacientes para lhes brindar apoio, dado o impacto e desgaste que gera ser cuidador primário em uma doença.

Palavras-chave: cuidadores primários cuidador; enfermagem; diabete mellitus tipo 2

\section{Introducción}

La diabetes mellitus se está convirtiendo rápidamente en la epidemia del siglo XXI y en un reto de salud global. Estimaciones de la Organización Mundial de la Salud indican que en el mundo, de 1995 a la fecha, casi se ha triplicado el número de personas que viven con diabetes, con una cifra actual estimada en más de 347 millones de personas con diabetes (1). De acuerdo con la Federación Internacional de Diabetes, China, India, Estados Unidos, Brasil, Rusia y México son —en ese orden - los países con mayor número de diabéticos. La diabetes es una enfermedad crónica de causas múltiples. En su etapa inicial no produce síntomas y cuando se detecta tardíamente y no se trata adecuadamente ocasiona complicaciones de salud graves como infarto del corazón, ceguera, falla renal, amputación de las extremidades inferiores y muerte prematura (2). Las enfermedades de carácter crónico se han convertido en una de las preocupaciones más importantes de la sociedad moderna, debido a que implica la no recuperación del estado de salud y una estadía permanente en los sistemas de salud; al mismo tiempo, el deterioro que generan en el enfermo causa que requieran de terceros que se hagan cargo de ellos. Una de esas enfermedades es la diabetes mellitus, muy estudiada desde el punto de vista médico (3). El diabético se enfrenta a todo un mundo nuevo de retos, entre ellos ser rotulado de enfermo para siempre, ver cómo se van degenerando sus funciones vitales y sociales, pasar varias horas en las instituciones de salud para controlar la enfermedad, sumado a las dificultades que encuentra para ser atendido en ciertas áreas (como las dentales y las quirúrgicas), por lo complicado que es la cicatrización de las heridas y las posibles hemorragias (4). El diagnóstico de enfermedad crónica trae consigo diferentes grados de dependencia para quien la padece, además de implicaciones psicológicas, sociales y espirituales que fundamentan la importancia de abordar la enfermedad más allá de sus implicaciones orgánicas (5).

En México, la transición demográfica y epidemiológica, así como el incremento de la esperanza de vida de la población, evidencia que la población está envejeciendo. Esto implica que el porcentaje de personas con alguna enfermedad crónico-degenerativa e incurable se incremente y que cada día aumente el número de pacientes que requieren un sistema de ayuda y apoyo, es decir, cuidados para cubrir las actividades que no pueden llevar a cabo por sí mismos; además de personas que se responsabilicen completamente de su cuidado (6). Por tal motivo, el cuidado familiar de personas con enfermedad crónica ha emergido durante los últimos años, dado que la diabetes se ha convertido como un importante problema de salud pública y social. Ante esta situación, la figura de cuidador primario se visualiza como aquella persona que, por parentesco o afinidad, asume las responsabilidades de cuidado de su ser querido, siendo partícipe de su asistencia, su experiencia vivida y su toma de decisiones (7).

Los cuidadores primarios se convierten en un componente esencial para favorecer la autonomía, satisfacer las necesidades de cuidado, contribuir en los procesos adaptativos de la enfermedad y favorecer respuestas positivas para la continuidad del cuidado (8).

En la actualidad se reconoce que el cuidado de los cuidadores informales es un elemento importante para la obtención y mantenimiento del control metabólico de las personas con diabetes; así mismo, favorecen la 
autonomía y satisfacen las necesidades de cuidado, y contribuyen en los procesos adaptativos de la enfermedad para propiciar respuestas positivas para la continuidad del cuidado.

Existen cuidadores informales, quienes se caracterizan por no haber recibido capacitación alguna sobre los cuidados que debe recibir la persona cuidada, por no ser remunerados por sus tareas y por brindar una atención sin límite de horarios. Este colectivo suele estar caracterizado por un elevado grado de compromiso hacia la tarea, determinado por las relaciones afectivas existentes entre cuidador y dependiente, sin una percepción económica (9). Por lo anterior, conocer el perfil de los cuidadores permitirá a los profesionales de enfermería establecer diferentes estrategias de salud que refuerce el conocimiento acerca de la enfermedad y mejore el estilo de vida de los pacientes, así como establecer intervenciones educativas para brindarles apoyo, dado el impacto y el desgaste que genera ser cuidador primario en una enfermedad que tiende a presentar complicaciones severas y demanda de atención de las necesidades básicas y emocionales, ya que no solo el paciente tiene pérdida, sino también la familia, y esto genera crisis en ambos.

La Facultad de Estudios Superiores (FES) Zaragoza de la Universidad Nacional Autónoma de México se localiza en el oriente de la Ciudad de México y cuenta con siete clínicas universitarias de atención a la salud (CUAS), las cuales brindan atención de enfermería, medicina, psicología y odontología a las comunidades aledañas.

Muchos de los cuidadores del presente estudio se caracterizan por llevar estilos de vida poco saludables, como el sedentarismo y la alta ingesta de lípidos y azúcares refinados. De ahí la importancia de implementar intervenciones de enfermería con base en el perfil de los cuidadores primarios, que permitan prevenir la diabetes. Por lo anterior, el objetivo de la presente investigación fue describir el perfil del cuidador primario del paciente diabético en dos comunidades aledañas a la FES Zaragoza.

\section{Material y métodos}

Se realizó un estudio transversal descriptivo entre agosto de 2017 y noviembre de 2018 con 38 cuidadores primarios de personas que padecen diabetes mellitus tipo 2, provenientes de dos CUAS: 17 de la CUAS Zaragoza y 21 de la CUAS Reforma. Esto a partir un censo que involucró a la totalidad de los cuidadores primarios que están con los pacientes diagnosticados con diabetes mellitus tipo 2 que forman parte de una investigación mayor y a quienes se les ha realizado un seguimiento desde 2016.

Los cuidadores primarios han sido visitados de manera regular por pasantes de Enfermería que realizan su servicio social en el proyecto. A cada cuidador primario se le solicitó un consentimiento informado que confirmara su participación en el proyecto. El tiempo estimado de aplicación del instrumento fue entre $15 \mathrm{y}$ 20 minutos. El instrumento que se utilizó se denominó Valoración para el cuidador primario. El instrumento contempló cinco componentes: características sociodemográficas, estado de salud del cuidador, interacción paciente-cuidador, conocimientos sobre la diabetes mellitus y valores del cuidador.

- Características sociodemográficas: edad, sexo, estado civil, escolaridad, ocupación, ingreso mensual.

Estado de salud del cuidador: padecimiento de alguna enfermedad, tiempo de evolución, si acude a revisión médica y la frecuencia con que acude, si cuenta con algún tipo de tratamiento.

Interacción paciente-cuidador primario: parentesco con el paciente, tiempo que le dedica al cuidado, tiempo que lleva cuidándolo, si existe otra persona que lo apoye en el cuidado y de qué manera lo apoyan y costo aproximado mensual que gasta en la enfermedad del paciente.

Conocimientos que tiene sobre diabetes mellitus tipo 2: donde se le hicieron 15 preguntas y se asignó un puntaje para determinar si era conocimiento deficiente (menor a 31 puntos), conocimiento regular ( 32 a 62 puntos), conocimiento bueno ( 63 a 79 puntos) o conocimiento excelente ( 80 puntos).

Valores del cuidador: los que considera importantes en el cuidado que proporcionan: la solidaridad, la responsabilidad, la empatía, el amor, la tolerancia y la dignidad del paciente. 
El instrumento cuenta con una validez de contenido que, de acuerdo con Hernández Sampieri et al. (10), se refiere al grado en que un instrumento refleja un dominio específico de contenido de los que se mide. Este instrumento fue sometido a una evaluación por expertos, quienes evaluaron de forma individual cada uno de los ítems del instrumento, con el fin de determinar su relevancia para la variable que se pretendía medir (11).

Para el análisis estadístico se utilizó el programa SPSS versión 22. A cada una de las variables de estudio se le aplicaron medidas de tendencia central y de dispersión, así como estadística descriptiva, donde se utilizan frecuencias y porcentajes, por lo que se presentan los resultados más relevantes. En el aspecto ético, el proyecto, al ser evaluado por una institución externa y ser financiado, se apega a los principios éticos, y normativamente, a la Ley General de Salud en Materia de Investigación para la Salud (título II, capítulo I, artículos 13, 14 y 16) de nuestro país, así como a los principios éticos contemplados en la Declaración de Helsinki y de la Conferencia Mundial de la armonización para las buenas prácticas.

\section{Resultados}

La edad promedio de los cuidadores es de 60 años \pm 15 años, con una edad mínima de 20 años y una máxima de 85 años. El sexo predominante de los cuidadores primarios es el femenino, representado por el 55,3\% (para un total de 21 cuidadoras); mientras que el sexo masculino está representado por el 44,7\% (es decir, 17 cuidadores).

El estado civil predominante de los cuidadores primarios es casado, representado por el $63,2 \%$ de los participantes, es decir, 24 cuidadores; entre tanto, los participantes con estado civil viudo y divorciado representan el menor porcentaje $(7,9 \%)$ equivalente a 3 cuidadores cada uno. La mayoría de los cuidadores primarios (76,3\%), es decir, 29 tienen entre 1 y 6 hijos. El 24,1\% de los cuidadores, es decir, 7 de ellos dan sustento económico a sus hijos.

Respecto a la ocupación de los cuidadores, la mayoría de ellos laboran en actividades remuneradas ( $42 \%)$, como carpintería, construcción, área de la energía eléctrica, comercio, transporte, gobierno, entre otros; mientras que el $40 \%$ desempeña labores en el hogar y únicamente el $18 \%$ se encuentra jubilado o pensionado.

En cuanto a la escolaridad de los cuidadores, se encontró que 17 (45\%) cuenta con primaria, 10 (26\%) con secundaria, 5 (13\%) con bachillerato, 1 (3\%) con estudios técnicos y 5 (13\%) con licenciatura. Sobre el ingreso económico mensual de los participantes, 7 de ellos percibe menos de 1500 pesos mexicanos al mes (18\%), seguido por quienes ganan entre 1501 y 2000 (21\%), 2001 y 2500 (18\%), 2501 y 3000 (21\%) y más de $3000(21 \%)$ pesos mexicanos.

La mayoría de los cuidadores cuenta con atención médica proporcionada por el Instituto Mexicano del Seguro Social (50\%). Únicamente 2 cuidadores no cuentan con atención médica, lo que representa el 5 $\%$ de estos. Quince cuidadores asisten al menos una vez al mes a una institución de salud, seguido por 13 que lo hacen de una a dos veces, 10 de ellos no asisten a visita médica.

El $68 \%$ de los cuidadores primarios padece alguna enfermedad ( $31 \%$ hipertensión arterial y $8 \%$ diabetes mellitus) con un tiempo de evolución de entre 2 y 5 años y el $60 \%$ cuenta con tratamiento farmacológico.

Con relación al parentesco de los cuidadores con los pacientes, se encontró que el $44 \%$ corresponde a las(os) esposas(os); el $36 \%$ es la madre o el padre, y solo el $5 \%$ son los hijos.

El tiempo que le dedican al cuidado de los pacientes en promedio son 12 horas. $\mathrm{El} 73 \%$ de los cuidadores cuenta con apoyo de sus familiares (hijos o hermanos) en el cuidado de los pacientes. Por comunidad se observan las características sociodemográficas de los cuidadores de ambas comunidades (tabla 1). 
TABLA 1.

Características sociodemográficas de los cuidadores primarios de ambas comunidades

\begin{tabular}{lcccc}
\hline \multirow{2}{*}{ Variables } & \multicolumn{2}{c}{ CUAS Zaragoza } & \multicolumn{2}{c}{ CUAS Reforma } \\
\cline { 2 - 5 } & $\mathbf{n}$. $^{\mathbf{0}}$ & $\%$ & $\mathbf{n}$ o $^{\mathbf{y}}$ & $\%$ \\
\hline Sexo & & & & \\
\hline Femenino & 7 & 41 & 14 & 67 \\
\hline Masculino & 10 & 59 & 7 & 33 \\
\hline Edad & & & & \\
\hline $20-42$ & 0 & 0 & 3 & 14 \\
\hline $43-65$ & 13 & 77 & 8 & 38 \\
\hline $66-80$ & 4 & 23 & 10 & 48 \\
\hline Estado civil & & & & \\
\hline Soltero & 4 & 23 & 4 & 19 \\
\hline Casado & 10 & 59 & 14 & 66 \\
\hline Viudo & 2 & 12 & 1 & 4 \\
\hline Divorciado & 1 & 6 & 2 & 9 \\
\hline Parentesco & & & & \\
\hline Esposo/a & 7 & 41 & 10 & 48 \\
\hline Madre/Padre & 4 & 23 & 10 & 48 \\
\hline Hijo(a) & 2 & 12 & 0 & 0 \\
\hline Hermana(o) & 3 & 18 & 0 & 0 \\
\hline Otros & 1 & 6 & 1 & 4 \\
\hline
\end{tabular}

\begin{tabular}{|c|c|c|c|c|}
\hline \multirow{2}{*}{ Variables } & \multicolumn{2}{|c|}{ CUAS Zaragoza } & \multicolumn{2}{|c|}{ CUAS Reforma } \\
\hline & n. ${ }^{\circ}$ & $\%$ & n.o & $\%$ \\
\hline \multicolumn{5}{|l|}{ Ingreso mensual } \\
\hline$<1500$ & 2 & 12 & 5 & 24 \\
\hline $1501-2000$ & 6 & 35 & 2 & 9 \\
\hline $2001-2500$ & 2 & 12 & 5 & 24 \\
\hline 2501-2999 & 3 & 18 & 5 & 24 \\
\hline$>3000$ & 4 & 23 & 4 & 19 \\
\hline \multicolumn{5}{|l|}{ Ocupación } \\
\hline Ama de casa & 7 & 41 & 8 & 38 \\
\hline Oficios & 4 & 23 & 3 & 14 \\
\hline Empleado & 0 & 0 & 6 & 29 \\
\hline Desempleado & 1 & 6 & 2 & 9 \\
\hline Jubilado/pensionado & 5 & 30 & 2 & 9 \\
\hline
\end{tabular}

Fuente: datos obtenidos del instrumento aplicado a los cuidadores primarios

En relación con el nivel de conocimientos sobre diabetes mellitus, se encontró que el $55 \%$ de los cuidadores en ambas comunidades cuenta con un conocimiento regular, y por clínica el $41 \%$ en Zaragoza y el $67 \%$ en Reforma y en ambas ningunas cuidadoras tiene un conocimiento excelente sobre la diabetes mellitus tipo 2 (tabla 2).

TABLA 2.

Nivel de conocimiento de los cuidadores primarios sobre la diabetes mellitus de su familiar

\begin{tabular}{lrrrr}
\hline Diabetes & Deficiente & Regular & Bueno & Excelente \\
\hline Conocimiento de los cuidadores primarios & $5(13 \%)$ & $21(55 \%)$ & $12(32 \%)$ & $0(0 \%)$ \\
\hline CUAS Zaragoza & $3(18 \%)$ & $7(41 \%)$ & $7(41 \%)$ & $0(0 \%)$ \\
\hline CUAS Reforma & $2(9 \%)$ & $14(67 \%)$ & $5(24 \%)$ & $0(0 \%)$ \\
\hline
\end{tabular}

Fuente: datos obtenidos del instrumento aplicado a los cuidadores primarios

Un aspecto que se ha explorado poco fueron los valores del cuidador, que considera importantes en el cuidado que proporcionan al paciente. Se encontró lo siguiente: un $90 \%$ manifestó tenerle respeto a su paciente, el $45 \%$ muestra solidaridad hacia el paciente en su enfermedad, el $67 \%$ manifestó que la responsabilidad es algo muy importante en el cuidado, así como el $49 \%$ mostró que la tolerancia hacia el paciente es importante en la cotidianidad con el paciente (tabla 3).

TABLA 3

Valores que manifestaron los cuidadores primarios en el cuidado hacia el paciente

\begin{tabular}{lll}
\hline Valores & n. $^{\circ}$ & \% \\
\hline Respeto & 34 & 90 \\
\hline Tolerancia & 19 & 49 \\
\hline Responsabilidad & 25 & 67 \\
\hline Solidaridad & 17 & 45
\end{tabular}

Fuente: datos obtenidos del instrumento aplicado a los cuidadores primarios

\section{Discusión}

En nuestro estudio, el perfil preponderante del cuidador principal coincide con el de otros autores, en los cuales la mujer es quien predomina en el cuidado $(12,13)$.

La edad promedio de los cuidadores fue de 65 años, mayor a lo reportado por Ambriz-López et al. (14) y por Ortiz-Claro et al. (13). Se encontró que el $67 \%$ vive bajo el mismo techo que el paciente frente al $72 \%$ de lo reportado por Quero Rufián et al. (15). 
En la relación del cuidador con el paciente se encontró que quienes los cuidan son las(os) esposas(os), en un $44 \%$. El $36 \%$ es la madre o padre, dato que difiere con Ambriz-López et al. (14), para quienes el 30,8 \% de los cuidadores era esposo(a) o pareja del paciente y el $24,8 \%$ era padre/madre.

Con relación a la ocupación de los cuidadores, en nuestro estudio se encontró que el $41 \%$ se dedica al hogar, lo que coincide con el estudio de Ortiz-Claro et al. (13), en el cual el $76 \%$ de los cuidadores son amas de casa. Las mujeres no solo asumen de forma mayoritaria el papel de cuidadoras principales; también se responsabilizan de tareas más pesadas y demandantes, y dedican más tiempo a cuidar que los hombres, lo que genera una clara inequidad de género.

En lo que respecta a redes de apoyo familiar, Lara-Palomino et al. (16) indicaron que el 76,7 \% comentó recibir ayuda por un cuidador secundario, y el 83,3\%, por otro familiar, coincidiendo con nuestros resultados, en los cuales el $73 \%$ de los cuidadores cuenta con apoyo de sus familiares (hijos o hermanos) en el cuidado de los pacientes.

El análisis de la dimensión del estado de salud del cuidador es un factor al que se le presta poca atención, tanto por la misma familia como por la propia institución. Sin embargo, se ha detectado (17) en el cuidador familiar la aparición de patologías de carácter crónico degenerativas como hipertensión, obesidad, cefalea, entre otras. Se puede establecer con esto la probabilidad de generar a futuro más adultos mayores enfermos dependientes con necesidades de un cuidador.

Otro de los resultados que se encontraron fueron las enfermedades crónicas asociadas en los cuidadores: el 68\% de los cuidadores primarios padece alguna enfermedad (31\% hipertensión arterial o $8 \%$ diabetes mellitus). Estos datos difieren de los resultados encontrados con el estudio de Rivera-Mora et al. (18) para quienes las enfermedades crónico-degenerativas presentes fueron hipertensión arterial en un $27 \%$, diabetes mellitus en un $18 \%$ y un $16 \%$ afecciones articulares.

En relación con los conocimientos que tienen los cuidadores sobre la diabetes mellitus se encontró que el $55 \%$ de los cuidadores en ambas comunidades cuentan con un conocimiento regular, lo cual no coincide con el estudio de Ramírez-Alvarado (19), en el que el 72 \% posee conocimientos sobre aspectos básicos de la DT2, control de glucemia y prevención de complicaciones (corte de uñas, alimentación y ejercicio).

Respecto a los valores que manifestaron los cuidadores primarios - un aspecto poco estudiado-, se encontró que la tolerancia y la responsabilidad estuvieron presentes en los cuidadores primarios y ello coincide con las autoras Eterovic et al. (20), quienes manifiestan que, de una u otra forma, quien asume la responsabilidad del cuidado informal en el hogar lo hace con un gran compromiso, mediado por la relación afectiva que lo une a la persona cuidada; pocas veces cuestionado sobre la propia habilidad de cuidado y disposición para asumir tal responsabilidad. La doctora Ngozi O. Nkongho, citada por Vega-Angarita et al., menciona que la paciencia es otro componente prioritario en la adquisición de habilidad de cuidado. Esta autora considera que "la paciencia es dar tiempo y espacio para la autoexpresión y exploración e incluye el grado de tolerancia ante la desorganización y problemas que limitan el crecimiento de sí mismo y de otros" (21).

Es importante resaltar que los resultados del perfil del cuidador obtenidos en este estudio permitirán al personal de enfermería rescatar aspectos importantes para el seguimiento en el hogar de la persona o familiar, que es el apoyo en varios aspectos (dieta, tratamiento o estilo de vida) de los enfermos de diabetes mellitus y así incidir en el retraso de complicaciones o reingreso en los hospitales. En relación con el componente de valores, los resultados muestran que aun cuando los cuidadores primarios aceptan la responsabilidad del cuidado, se tienen que implementar intervenciones de enfermería en diferentes ámbitos para evitar la sobrecarga, para fomentar el autocuidado, disminuir los sentimientos de intranquilidad que a veces se manifiestan en los cuidadores, pero, sobre todo, ayudar a que los cuidadores estén mejor preparados para los retos que implica un enfermo de diabetes mellitus en el hogar. 


\section{Conclusiones}

La familia desempeña un papel importante como fuente de protección y apoyo en los pacientes con enfermedades crónico-degenerativas; en nuestro caso, pacientes con diabetes mellitus. Este aspecto necesita una mayor atención por parte del sistema de salud, especialmente de los profesionales de la enfermería, ya que la atención de estos pacientes puede repercutir principalmente en los cuidadores en la reducción de su tiempo libre, en ocasiones en las relaciones sociales (hasta llegar a la fatiga que puede ocasionar una sobrecarga) con el consiguiente deterioro de la salud física y psicológica.

Un aspecto relevante que se encontró en el estudio fue que el perfil del cuidador es muy similar en ambas comunidades. Se observó que predominó el sexo femenino en el cuidado de los pacientes. Como lo refiere la literatura, los adultos tienden a cuidar más que los jóvenes. Las personas casadas son las que destacaron en el estudio y son las que atienden a sus familiares. Los conocimientos que tienen los cuidadores sobre la diabetes mellitus son regulares, lo que indica la importancia del trabajo en educación para la salud por parte de enfermería en los ámbitos hospitalario y domiciliario, a través de un programa de apoyo sobre acciones preventivas hacia los cuidadores, que les permita reforzar el conocimiento acerca de la enfermedad y así mejorar el estilo de vida de los pacientes para brindarles un apoyo, dado el impacto y el desgaste que genera ser cuidador primario en una enfermedad.

\section{Referencias}

1. Organización Mundial de la Salud. Diabetes Nota descriptiva 312. Washington: OMS; 2012 [citado 2019 ene 2]. Disponible en: http://www.who.int/mediacentre/factsheets/fs312/es/index.html

2. Hernández-Ávila M, Gutiérrez JP, Reynoso-Noverón N. Diabetes mellitus en México: el estado de la epidemia. Salud Pública Mex. 2013;55(2):129-136. http://dx.doi.org/10.21149/spm.v55s2.5108

3. Venegas-Bustos BC. Habilidad del cuidador y funcionalidad de la persona cuidada. Aquichan [internet]. 2006;6(1):137-47. Disponible en: https://aquichan.unisabana.edu.co/index.php/aquichan/article/view/87

4. Moraga PR. Estudio cualitativo sobre la experiencia subjetiva de la enfermedad en diabéticos. Rev Chil Salud Pública [internet]. 2005;9(3):162-8. Disponible en: https://revistasaludpublica.uchile.cl/index.php/RCSP/article/vie $\mathrm{w} / 20158$

5. Vinaccia $S$, Quiceno M. Calidad de vida relacionada con la salud y enfermedad crónica: estudios colombianos. Acta. Colomb. Psicol. 2012;6(1):123-36. http://dx.doi.org/10.14718/ACP.2017.20.1.4

6. Guerrero-Nava JA et al. El cuidador primario, su paciente terminal y la enfermería. Rev Enferm Inst Mex Seguro Soc. 2016;24(2):91-8.

7. Ruiz-Robledillo N, Moya-Albiol L. El cuidado informal: una visión actual. Rev Electrón Motiv Emoc [internet]. 2012;1:22-30. Disponible en: http://reme.uji.es/reme/3-albiol_pp_22-30.pdf

8. Pinzón-Rocha M, Aponte-Garzón L, Hernández-Páez R. Experiencia de los cuidadores informales en el manejo de la diabetes mellitus tipo II. Orinoquia. 2013;17:241-51. https://doi.org/10.22579/20112629.22

9. Comas D’argemir Dolors. Los cuidados de larga duración y el cuarto pilar del sistema de bienestar. Rev Antropol Soc. 2015;24:375-404. https://doi.org/10.5209/rev_RASO.2015.v24.50663

10. Hernández-Sampieri R, Fernández-Collado C, Baptista-Lucio P. Metodología de la investigación. 4. ${ }^{a}$ ed. México: McGraw Hill; 2008. pp. 278-9.

11. Chacón S, Pérez JA, Holgado FP, Lara Á. Evaluación de la calidad universitaria: validez de contenido. Psicothema. 2001;13(2):294-301.

12. Martínez-Cepero FE. Necesidades de aprendizaje del cuidador principal para el tratamiento del anciano con demencia tipo Alzheimer. Rev Cubana Enferm [internet]. 2009 [citado 2018 nov 13];25:(3-4). Disponible en: http://scielo.sld.cu/pdf/enf/v25n3-4/enf06309.pdf 
13. Ortiz-Claro YG, Lindarte-Clavijo AA, Jiménez-Sepúlveda MA, Vega-Angarita OM. Características sociodemográficas asociadas a la sobrecarga de los cuidadores de pacientes diabéticos en Cúcuta. Revista Cuidarte. 2013;4(1):459-66. https://doi.org/10.15649/cuidarte.v4i1.5

14. Ambriz-López MC, Villalobos-Arámbula LM, Gallegos-Torres RM, Xeque-Morales AS, Hernández-Castañón MA, Palomé-Vega G. Perfil del cuidador principal y su relación con el cuidado de enfermería. Rev Enferm Inst Mex Seguro Soc [internet]. 2014;22(3):123-9. Disponible en: https://www.medigraphic.com/pdfs/enfermeria imss/eim-2014/eim143b.pdf

15. Quero-Rufián A, Briones-Gómez R, Prieto-Rodríguez MA, Navarro López A, Pascual-Martínez N, Guerrero-Ruiz C. Perfil y realidad social de los cuidadores familiares en un hospital de traumatología y rehabilitación. Nure Investigación [internet]. 2004;(9):1-14. Disponible en: https://www.nureinvestigacion.es/OJS/index.php/nur e/article/view/192/0

16. Lara-Palomino G, González Pedraza Avilés A, Blanco-Loyola LA. Perfil del cuidador: sobrecarga y apoyo familiar e institucional del cuidador primario en el primer nivel de atención. Rev Esp Med-Quir [internet]. 2008;13(4):159-66. Disponible en: https://www.medigraphic.com/pdfs/quirurgicas/rmq-2008/rmq084c.pdf

17. Compean GJM, Silerio VJ, Castillo DR, Parra DML. Perfil y sobrecarga del cuidador primario del paciente adulto mayor con afecciones neurológicas [internet]. Rev Conamed. 2008;13(Suppl 1):S17-21. Disponible en: https://imbiomed.com.mx/1/1/articulos.php?method=showDetail\&id_articulo=48629\&id_seccion=69 9\&id_ejemplar=4928\&id_revista $=41$

18. Rivera-Mora H, Dávila-Mendoza R, González Pedraza-Avilés A. Calidad de vida de los cuidadores primarios de pacientes geriátricos de la Clínica de Medicina Familiar Dr. Ignacio Chávez. Rev Esp Med-Quir. 2011;16(1):27-32.

19. Ramírez-Alvarado GG. Conocimientos y habilidades de los cuidadores de adultos con diabetes tipo 2 [tesis de maestría]. Bogotá: Universidad Nacional de Colombia; 2012.

20. Eterovic-Díaz C, Mendoza-Parra S, Sáez-Carrillo K. Habilidad de cuidado y nivel de sobrecarga en cuidadoras/es informales de personas dependientes. Enferm Global [internet]. 2015;(38):235-48. Disponible en: http://sciel o.isciii.es/pdf/eg/v14n38/administracion4.pdf

21. Vega-Angarita O, Mendoza-Tarazona MK, Ureña-Molina M del P, Villamil Santander WA. Efecto de un programa educativo en la habilidad de cuidado de los cuidadores familiares de personas en situación crónica de enfermedad. Rev Cienc Cuidado. 2008;1(5):5-19. https://doi.org/10.22463/17949831.927

\section{Notas}

* Artículo original de investigación

Conflictos de interés: las autoras declaran no tener conflictos de interés.

\section{Licencia Creative Commons CC BY 4.0}

Como citar este artículo: González Velásquez MS, Crespo Knopfler S. Perfil del cuidador primario en pacientes diabéticos en dos comunidades. Investig Enferm Imagen Desarr. 2020;22. https://doi.org/10.11144/Javeri ana.ie22.pcpd

Financiación: Este trabajo se deriva del proyecto "Atención de enfermería en comunidad a pacientes con enfermedades crónicas no transmisibles”, financiado por la Fundación Gonzalo Río Arronte y la Universidad Nacional Autónoma de México, clave 43226-2936-9-XI-15.

Limitaciones: Una de las limitaciones que se tuvo en este estudio fue el tamaño de la muestra, ya que ello no nos ha permitido utilizar una estadística inferencial para establecer asociaciones o causalidades que establezcan generalizaciones. 